\title{
0735-1933(94)00045-X
}

\section{NATURNAL CONVECTION OF A DARCIAN FLUID ABOUT A WAVY CONE}

\author{
Ioan Pop \\ Faculty of Mathematics \\ University of Cluj, R-3400 Cluj, CP253, Romania \\ Tsung-Yen $\mathrm{Na}$ \\ Department of Mechanical Engineering \\ University of Michigan - Dearborn, Dearborn, MI, 48128, U.S.A.
}

(Communicated by J.P. Hartnett and W.J. Minkowycz)

\begin{abstract}
The results of a study of natural convection along an isothermal wavy cone embedded in a fluid-saturated porous medium are presented. The boundary layer under consideration is cencerned with the regime where the Darcy-Rayleigh number $R a$ is very large. It is assumed that the surface waves have $O(1)$ amplitude and wavelength. The boundary layer equations are solved numerically using the Keller-box method. Detailed results of the effect of the sinusoidal waves on the heat flux on the wall are given.
\end{abstract}

\section{Introduction}

Natural convection from wavy surfaces is of importance in several heat transfer devices such as flat-plate solar collectors and flat-plate condensers in refrigerators. The presence of roughness elements disturbs the flow past surfaces and alters the heat transfer rate. Most of the reported surveys on natural convection in a porous medium deal with cases in which surfaces are uniform, see Nield and Bejan[1]. The interest of research in the case of natural convection from wavy surfaces has surged during recent years, see references [2-6]. 
In the present paper, a theoretical study of the natual convection along a vertical cone with transverse curvature and sinusoidal undulations embedded in a fluid-saturated porous medium is reported. Of primary relevance to this work are the papers by Rees and Pop $[5,6]$ in which the authors reported numerical solutions for the problem of natural convection along a vertical wavy surface in a porous medium. To the best of our knowledge, natural convection along a cone with transverse undulations in a porous medium has not been reported prior to the present analysis.

\section{$\underline{\text { Analysis }}$}

Consider a vertical cone with transverse sinusoidal undulations embedded in a fluid-saturated porous medium, as illustrated in Fig.1. In particular, we assume that the surface profile is given by

$$
\bar{y}_{w}=\bar{\sigma}(\bar{x})=\bar{a} \sin \left(\frac{\pi \bar{x}}{\ell}\right)
$$

where $\bar{a}$ is the amplitude of the transverse surface wave and $\ell$ is the characteristic length of the wave. The surface of the cone is held at a constant temperature $T_{w}$, which is greater than the temperature of the ambient fluid $T_{\infty}$

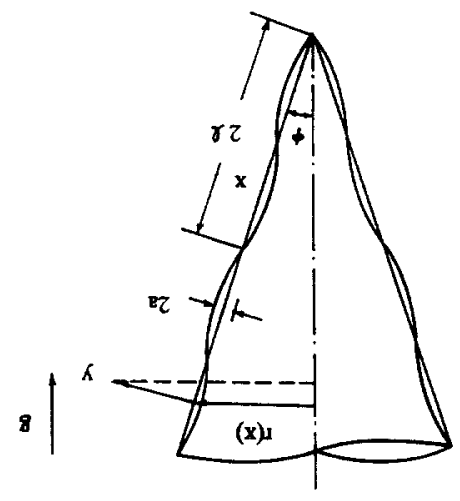

FIG.1

Physical Model and Coordinate System

The non-dimensional equations governing natural convection from the wavy cone can be written under the Darcy-Boussinesq approximation as 


$$
\begin{gathered}
\frac{\partial(r u)}{\partial x}+\frac{\partial(r v)}{\partial y}=0 \\
\frac{\partial u}{\partial y}-\frac{\partial v}{\partial x}=R a\left\{\frac{\partial \theta}{\partial y}+\frac{\partial \theta}{\partial x} \tan \Phi\right\} \\
\frac{\partial^{2} \theta}{\partial x^{2}}+\frac{\partial^{2} \theta}{\partial y^{2}}=u \frac{\partial \theta}{\partial x}+v \frac{\partial \theta}{\partial y}
\end{gathered}
$$

where $(x, y)$ are the Cartesian coordinates, $(u, v)$ are the velocity components along $(x, y)$-axes, $R a$ is the Darcy-Rayleigh number, $\Phi$ and $r$ are the half angle and the local radius of the smooth surface of the cone, where $r$ is given by

$$
r=x \sin \Phi
$$

The dimensionless variables are defined by

$$
\begin{gathered}
x=\frac{\bar{x}}{\ell}, y=\frac{\bar{y}}{\ell}, \quad \psi=\frac{\bar{\psi}}{\alpha}, r=\frac{\bar{r}}{\ell} \cdot \theta=\frac{T-T_{\infty}}{T_{w}-T_{\infty}}, \\
\sigma=\frac{\bar{\sigma}}{\ell}, \quad a=\frac{\bar{a}}{\ell}, \quad R a=\frac{g \beta K\left(T_{w}-T_{\infty}\right) \ell \cos \Phi}{\alpha \nu}
\end{gathered}
$$

where $T$ is the temperature, $g$ is the acceleration due to gravity, $\beta$ is the expansion coefficient of the fluid, $\nu$ is the kinematic viscosity of the fluid, $K$ and $\alpha$ are the permeability and thermal diffusivity of the fluid - saturated porous medium.

Introducing the stream function $\psi$ defined by

$$
u=\frac{1}{r} \frac{\partial \psi}{\partial y} ; v=-\frac{1}{r} \frac{\partial \psi}{\partial x}
$$

where the equation of continuity is satisfied identically and Eqs.(3) and (4) become

$$
\frac{1}{r}\left\{\frac{\partial^{2} \psi}{\partial x^{2}}+\frac{\partial^{2} \psi}{\partial y^{2}}-\frac{r_{x}}{r}\left(\frac{\partial \psi}{\partial x}+\frac{\partial \psi}{\partial y}\right)\right\}=R a\left\{\frac{\partial \theta}{\partial y}+\frac{\partial \theta}{\partial x} \tan \Phi\right\}
$$




$$
\frac{\partial^{2} \theta}{\partial x^{2}}+\frac{\partial^{2} \theta}{\partial y^{2}}=\frac{1}{r}\left\{\frac{\partial \psi}{\partial y} \frac{\partial \theta}{\partial x}-\frac{\partial \psi}{\partial x} \frac{\partial \theta}{\partial y}\right\}
$$

where the subscript $x$ denotes differentiation with respect to $x$. The boundary conditions of Eqs.(8) and (9) are

$$
\begin{gathered}
\psi=0, \theta=1 \quad \text { on } y=\sigma(x)=a \sin (\pi x) \\
\frac{\partial \psi}{\partial y}=0, \theta=0 \quad \text { as } \quad y \rightarrow \infty
\end{gathered}
$$

We now assume that the Darcy-Rayleigh number $R a$ is large so that natural convection takes place within a boundary layer whose cross-stream width is substantially smaller than the amplitude $a$ of the wavy surface of the cone. Accordingly, we define new variables by substracting out the effect of the surface waves and then introduce the usual boundary layer variables defined as

$$
x=\tilde{x}, y=R a^{-\frac{1}{2}} \tilde{y}+\sigma(\tilde{x}), \quad \psi=R a^{\frac{1}{2}} \tilde{\psi}
$$

By substituting Eq.(12) into Eqs.(8) and (9), we get

$$
\begin{gathered}
\frac{1}{r}\left\{\left(1+\sigma_{\tilde{x}}^{2}\right) \frac{\partial^{2} \tilde{\psi}}{\partial \tilde{y}^{2}}-2 \sigma_{\tilde{x}} R a^{-\frac{1}{2}} \frac{\partial^{2} \tilde{\psi}}{\partial \tilde{x} \partial \tilde{y}}-\sigma_{\tilde{x} \tilde{x}} R a^{-\frac{1}{2}} \frac{\partial \tilde{\psi}}{\partial \tilde{y}}\right. \\
\left.+R a^{-\frac{1}{2}} \frac{\partial^{2} \tilde{\psi}}{\partial \tilde{x}^{2}}-\frac{r_{\tilde{x}}}{r}\left(1-\sigma_{\tilde{x}}\right) R a^{-\frac{1}{2}} \frac{\partial \tilde{\psi}}{\partial \tilde{y}}-\frac{r_{\tilde{x}}}{r} R a^{-1} \frac{\partial \tilde{\psi}}{\partial \tilde{x}}\right\} \\
=\left(1-\sigma_{\tilde{x}} \tan \Phi\right) \frac{\partial \theta}{\partial \tilde{y}}+R a^{-\frac{1}{2}} \tan \Phi \frac{\partial \theta}{\partial \tilde{x}} \\
\left(1+\sigma_{\tilde{x}}^{2}\right) \frac{\partial^{2} \theta}{\partial \tilde{y}^{2}}-2 \sigma_{\tilde{x}} R a^{-\frac{1}{2}} \frac{\partial^{2} \theta}{\partial \tilde{x} \partial \tilde{y}}-\sigma_{\tilde{x} \tilde{x}} R a^{-\frac{1}{2}} \frac{\partial \theta}{\partial \tilde{y}} \\
+R a^{-1} \frac{\partial^{2} \theta}{\partial \tilde{x}^{2}}=\frac{1}{r}\left\{\frac{\partial \tilde{\psi}}{\partial \tilde{y}} \frac{\partial \theta}{\partial \tilde{x}}-\frac{\partial \tilde{\psi}}{\partial \tilde{x}} \frac{\partial \theta}{\partial \tilde{y}}\right\}
\end{gathered}
$$


and the boundary conditions are now

$$
\begin{gathered}
\tilde{y}=0: \tilde{\psi}=0, \quad \theta=1 \\
\tilde{y}=\infty: \frac{\partial \tilde{\psi}}{\partial \tilde{y}}=0, \quad \theta=0
\end{gathered}
$$

If we formally let $R a$ approach to infinity, Eqs.(13) and (14) reduce to the following boundary layer equations for the problem under consideration,

$$
\begin{gathered}
\frac{1+\sigma_{\tilde{x}}^{2}}{r} \frac{\partial^{2} \tilde{\psi}}{\partial \tilde{y}^{2}}=\left(1-\sigma_{\tilde{x}} \tan \Phi\right) \frac{\partial \theta}{\partial \tilde{y}} \\
\left(1+\sigma_{\tilde{x}}^{2}\right) \frac{\partial^{2} \theta}{\partial \tilde{y}^{2}}=\frac{1}{r}\left\{\frac{\partial \tilde{\psi}}{\partial \tilde{y}} \frac{\partial \theta}{\partial \tilde{x}}-\frac{\partial \tilde{\psi}}{\partial \tilde{x}} \frac{\partial \theta}{\partial \tilde{y}}\right\}
\end{gathered}
$$

and the boundary conditions are those given by Eqs.(15) and (16). It should be mentioned that there was tacitly assumed that the surface waves have $O(1)$ amplitude and wavelength.

We shall now proceed to obtain numerical solutions of Eqs.(17) and (18). Thus, we assume that the solution is written as

$$
\tilde{\psi}=r \xi^{\frac{1}{2}} f(\xi, \eta), \theta=g(\xi, \eta)
$$

where

$$
\xi=\tilde{x} \quad, \quad \eta=\frac{\tilde{y}}{\xi^{\frac{1}{2}}\left(1+\sigma_{\xi}^{2}\right)}
$$

and $r$ is defined by Eq.(5). Introducing Eqs.(19) and (20) into Eqs.(17) and (18) gives

$$
\begin{gathered}
\frac{\partial^{2} f}{\partial \eta^{2}}=\left\{1-\sigma_{\xi} \tan \Phi\right\} \frac{\partial g}{\partial \eta} \\
\frac{\partial^{2} g}{\partial \eta^{2}}+\frac{3}{2} f \frac{\partial g}{\partial \eta}=\xi\left\{\frac{\partial f}{\partial \eta} \frac{\partial g}{\partial \xi}-\frac{\partial f}{\partial \xi} \frac{\partial g}{\partial \eta}\right\}
\end{gathered}
$$

which are subject to the boundary conditions 


$$
\begin{aligned}
\eta=0 & : f(\xi, 0)=0, g(\xi, 0)=1 \\
\eta=\infty & : \quad \frac{\partial f(\xi, \infty)}{\partial \eta}=0, g(\xi, \infty)=0
\end{aligned}
$$

It is worthy of mentioning that in the case of a smooth cone $(a=0)$, Eqs.(21) and (22) reduce to ordinary differential equations, which are identical with those found by Cheng et. al. [7].

An important physical quantity for this problem is the local Nusselt number defined as

$$
N u=\frac{\bar{x} q_{w}}{k\left(T_{w}-T_{\infty}\right)}
$$

where $k$ is the thermal conductivity of the fluid-saturated porous medium and $q_{w}$ is the heat flux at the wall, which is defined by

$$
q_{w}=-k \vec{n} \cdot \nabla T
$$

and

$$
\vec{n}\left\{-\frac{\sigma_{\tilde{x}}}{\left(1+\sigma_{\tilde{x}}^{2}\right)^{1 / 2}}, \frac{1}{\left(1+\sigma_{\tilde{x}}^{2}\right)^{1 / 2}},\right\}
$$

is the unit vector normal to the wavy surface of the cone. Using Eqs.(6) and (12), one can show that $N u$ can be expressed as

$$
\frac{N u}{R a^{\frac{1}{2}}}=\frac{\xi^{\frac{1}{2}}}{\left(1+\sigma_{\xi}^{2}\right)^{\frac{1}{2}}}\left\{-\frac{\partial g}{\partial \eta}\right\}_{\eta=0}
$$

\section{$\underline{\text { Result and Discussions }}$}

The boundary layer equations, Eqs.(21) and (22), along with the boundary conditions, Eqs.(23) and (24), were solved numerically by means of the Keller-box method (see references[8,9,10]) for some representative values of the wavy amplitude $a$ and half angle $\Phi$. The variation of Nusselt number 
with $\xi$ for $a=0$ (smooth cone), $0.2,0.4$ and $\Phi=10^{\circ}, 15^{\circ}, 20^{\circ}, 30^{\circ}$ are shown inFigs. 2 to 4 .

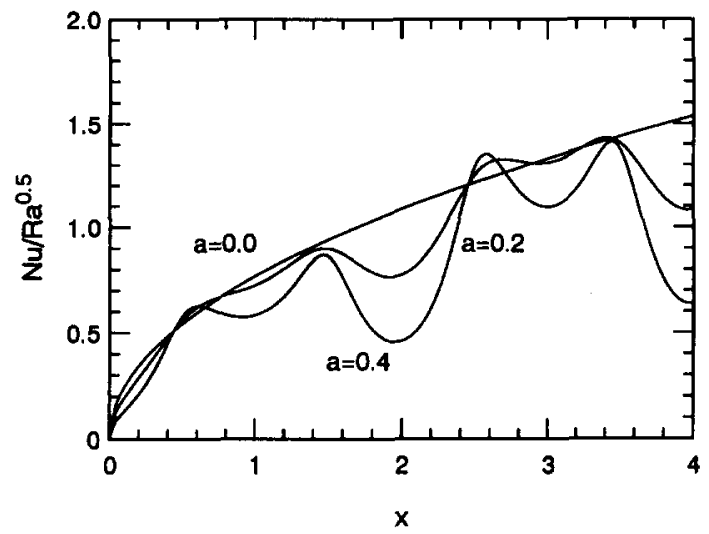

FIG.2

Variation of Local Nusselt Number with $x$ for $\Phi=15^{\circ}$

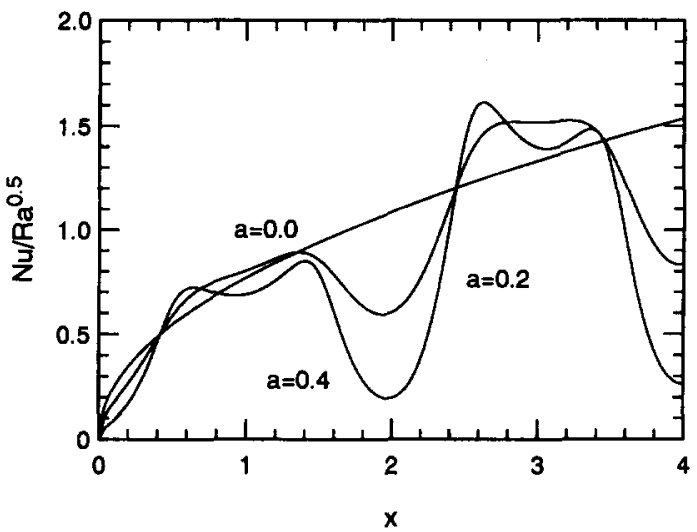

FIG.3

Variation of Local Nusselt Number with $x$ for $\Phi=30^{\circ}$

Fig. 2 shows the Nusselt number ratio defined on the left-side of Eq.(28) as a function of $\xi$. The effect of the amplitude $a$, on the average, is seen 
to decrease the heat transfer rate as compared with the limiting case of a smooth cone. The range of $x$ in the figure is from 0 to 4 which corresponds to two complete cycles of the undulations as shown in Fig.1. The raise and fall of the curves is seen to follow the change of the surface contour. Consider one complete cycle, i.e., for $x$ from 0 to 2. The rate of heat transfer is seen to increase for the first quarter of the cycle (from $x \approx 0$ to $x \approx 0.5$ ) and then decreases in the second quarter (from $x \approx 0.5$ to $x \approx 1$ ). From $x \approx 1$. to $x \approx 1.5$ (i.e., the third quarter), the heat transfer rate increases whereas in the last quarter (from $x \approx 1.5$ to $x \approx 2$ ), a larger decrease in the heat transfer rate resulted. The cycle will repeat itself as $x$ is advanced by another cycle. The results represent the nonlinear coupling of the change in fluid velocity and orientation of the gravitation. Fig. 3 shows the same conclusion can be obtained for anothe half cone angle, namely, $\Phi=30^{\circ}$. Fig. 4 show the effect of the cone angle $\Phi$ on the rate of heat transfer. As expected, the effects are more pronounced for larger half cone angles.

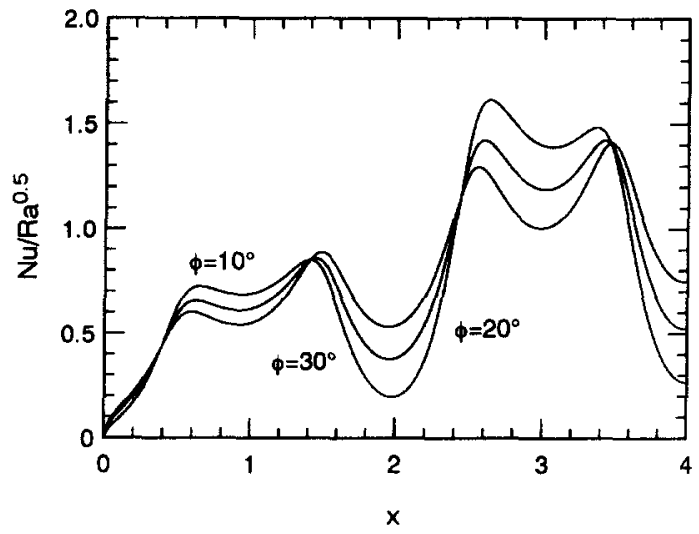

FIG.4

Variation of Local Nusselt Number with $x$ for $a=0.4$

\section{References}

[1] D.A.Nield and A.Bejan, Convection in Porous Media, Springer, Berlin, 1992.

[2] L.S.Yao, J. Heat Transfer, 105 , 465 (1983). 
[3] S. Ghosh Moulic and L.S.Yao, J. of Heat Transfer, 111 , 974(1989).

[4] S. Ghosh Moulic and L.S.Yao, J. of Heat Transfer, 111, 1106(1989).

[5] D.A.S.Rees and I.Pop, J. Heat Transfer, in press, 1994.

[6] D.A.S.Rees and I.Pop, J. Heat Transfer, accepted, 1994.

[7] P.Cheng, T.T.Lee and I.Pop, Int'l Comm. Heat and Mass Transfer, $12,705(1985)$.

[8] T.Y.Na, Appl. Sci. Res.,33 , 519(1978).

[9] T.Y.Na and I.Pop, Int'l J. Eng. Sci., 21 , 517(1983).

[10] T.Y.Na, Computational Methods in Engineering Boundary Value Problems, Academic Press, 1979.

Received June 22, 1994 